\title{
Título del Libro: La investigación cuantitativa \\ Capítulo 2: Planteamiento del problema y marco teórico en la investigación cuantitativa
}

ISBN: 978-980-18-1527-3

Depósito Legal: B02020000056

Edición: Primera Edición

\section{Editorial: AutanaBooks}

\section{Fecha de Publicación:}

Autores: Calizaya José Manuel, Bellido Medina Rildo Santos, Alemán Vilca Yaneth, Morales Palao Blanca, Monzón Álvarez Gloria Isabel, Ceballos Bejarano Ferdinand Eddington.

\section{INFORMACIÓN DE LOS AUTORES}

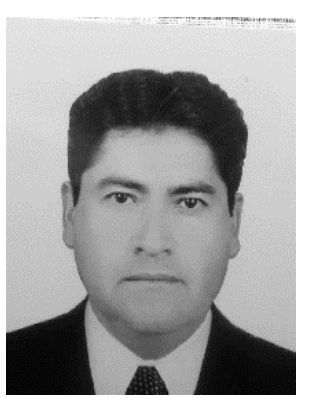

Calizaya López, José Manuel, Doctor en Ciencias de la Educación, Magíster en Ciencias con Mención en Gestión Social - Desarrollo Sostenible y Licenciado en Trabajo Social. Docente Principal y Director del Departamento Académico de Trabajo Social, Docente investigador UNSA- INVESTIGA y miembro del Instituto de Investigaciones Sociales de la Universidad Nacional de San Agustín, Arequipa, Perú.

ORCID: https:/ / orcid.org/0000-0001-6221-0909

Correo: jcalizayal@unsa.edu.pe

Afiliación institucional: Universidad Nacional de San Agustín de Arequipa

País: Perú

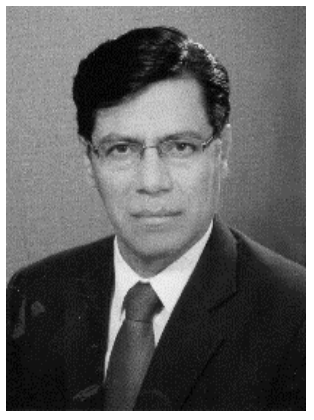

Bellido Medina, Rildo Santos, Doctor en Psicología y Magíster en Gerencia Estratégica de Recursos Humanos. Docente auxiliar en el programa de estudios de Relaciones Industriales, Docente investigador UNSA- INVESTIGA y miembro del Instituto de Investigaciones Sociales de la Universidad Nacional de San Agustín, Arequipa, Perú.

ORCID: https:/ / orcid.org/0000-0002-8699-3490

Correo: rbellidome@unsa.edu.pe

Afiliación institucional: Universidad Nacional de San Agustín de Arequipa

País: Perú 


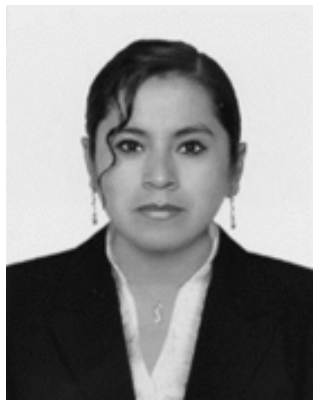

Alemán Vilca, Yaneth, Doctora en Administración (DBA), Maestra en Ciencias, con mención en Gerencia Social y de Recursos Humanos. Asesora de Proyectos de Investigación relacionados a la salud pública y mental. Docente de la Escuela Profesional de Trabajo Social de la Universidad Nacional de San Agustín.

ORCID: https:/ / orcid.org/0000-0002-9820-6036

Correo: yaleman@unsa.edu.pe

Afiliación institucional: Universidad Nacional de San Agustín de Arequipa

País: Perú

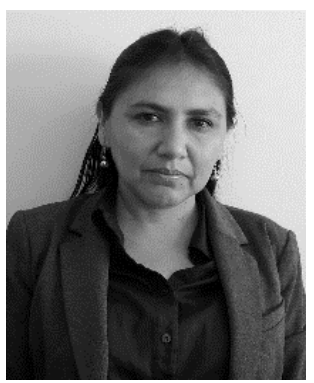

Morales Palao, Blanca, Magíster en Ciencias, Relaciones Industriales con mención en Gerencia Estratégica de Recursos Humanos, Licenciada en Ciencias de la Comunicación, Especialidad Relaciones Públicas, Diplomado en Recursos Humanos, Docente Auxiliar del programa de estudios de Ciencias de la Comunicación y miembro del Instituto de Investigaciones Sociales de la Universidad Nacional de San Agustín, Arequipa, Perú

ORCID: https:/ / orcid.org/0000-0001-9204-3400

Correo:bmorales@unsa.edu.pe

Afiliación institucional: Universidad Nacional de San Agustín de Arequipa

País: Perú

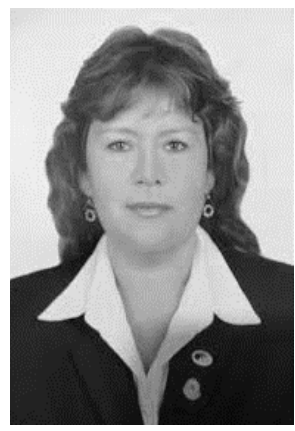

Monzón Álvarez, Gloria Isabel, Doctora en Administración (DBA), Magister en Estrategias de Desarrollo y Políticas Sociales, Licenciada en Trabajo Social, integrante de la Unidad de Investigación de la Facultad de Ciencias Histórico-Sociales. Experta en ejecución de Programas de Bienestar Social, Promoción de la Salud y Políticas del Sistema Integral de Salud Pública. Docente de la Escuela Profesional de Trabajo Social de la Universidad Nacional de San Agustín.

ORCID: https:/ / orcid.org/0000-0001-8712-5188

Correo:gmonzon@unsa.edu.pe

Afiliación institucional: Universidad Nacional de San Agustín de Arequipa

País: Perú

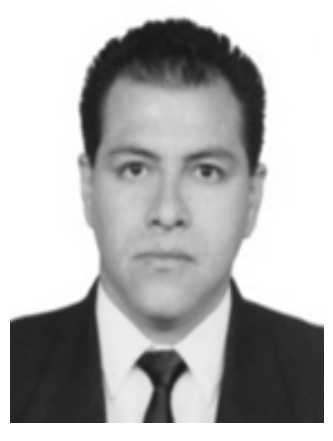

Ceballos Bejarano, Ferdinand Eddington, Licenciado en Matemática, Magíster en Finanzas y Administración de Negocios, Doctor en Ciencias Empresariales. Docente auxiliar en el programa de estudios de Administración y Finanzas, y miembro del Instituto de Investigaciones Sociales de la Universidad Nacional de San Agustín, Arequipa, Perú.

ORCID: https:/ / orcid.org/0000-0003-2867-2397

Correo: fceballos@unsa.edu.pe

Afiliación institucional: Universidad Nacional de San Agustín de Arequipa

País: Perú 
Tabla de contenido

Título del Libro: La investigación cuantitativa.

Capítulo 2: Planteamiento del problema y marco teórico en la investigación cuantitativa. 1

Introducción 3

Implicaciones del problema en la investigación cuantitativa. .5

Aspectos a considerar para el planteamiento del problema en la investigación cuantitativa

Formulación de objetivos 8

Las preguntas de investigación 9

Ejemplos de objetivos de investigación.

Ejemplos de preguntas de investigación.

Marco teórico en la Investigación Cuantitativa.

Elementos del marco teórico

La revisión bibliográfica.

Referencias.

\section{Prólogo}

En este libro se exponen los conceptos fundamentales de la investigación cuantitativa, y se consideran para ello la experiencia de sus autores, su trayectoria por la comunidad científica y la validación de otros profesionales del área de investigación, se consideran entonces los criterios para encaminar una investigación cuantitativa, así como cada uno de los pasos necesarios para que los proyectos cuantitativos se lleven a cabo con éxito. En este trabajo se muestra el primer capítulo, que comprende los aspectos teóricos de la investigación cuantitativa, la forma correcta de formular el problema y los elementos que hacen posible una estructura adecuada del problema en la investigación cuantitativa.

\section{Introducción.}

La investigación cuantitativa es una rama de la ciencia que es muy significativa para la mayoría de los proyectos de investigación, en las áreas de ingeniería por ejemplo resulta de gran relevancia, pero actualmente es aplicada en una infinidad de áreas profesionales, especialmente en las áreas sociales ha tomado gran auge en los últimos años, y es utilizada en todas las profesiones que requieren de un manejo de datos numéricos importantes y sus resultados dependen de información cuantificable. 
La base fundamental de las investigaciones cuantitativas radica en la necesidad de demostrar o contradecir una hipótesis y suele sustentarse con la recolección de encuestas. Sin embargo no necesariamente es así, y pueden surgir otras variantes que dependerán del tipo de estudio, ya que también pueden ser experimentos que relacionan variables para evaluar la causa y efecto de la independencia o interdependencia de las mismas ante un problema. Las principales características de la investigación cuantitativa incluyen recopilación de datos, la cual debe realizarse de forma sistemática y organizada, siguiendo un proceso riguroso que asegure la confiabilidad de los datos. Los números suelen reflejar la realidad de las situaciones, por eso deben mantenerse cuidadosamente sin alterar los valores que puedan modificar los resultados de la investigación.

Otra característica es la rapidez en la recolección de datos, pues gran parte de las investigaciones cuantitativas cuentan con una encuesta o cuestionario, que resulta de gran utilidad al momento de la toma de datos. Estas encuestas suelen procesarse de manera sencilla y rápida con software específicos que facilitan el procesado numérico de la información. Además la visualización de resultados a través de gráficos resulta por lo general muy fácil de comprender para los analistas y lectores de la investigación cuantitativa.

La investigación cuantitativa es fundamentalmente numérica, y por ende está asociada la estadística. Es posible entonces mencionar dos tipos de investigación cuantitativa:

Investigación cuantitativa a través de encuestas: este tipo de investigación se realiza a través de diferentes medios, que incluyen la recopilación de encuestas en formularios impresos, a través de medios digitales, correo electrónico, redes sociales o medios electrónicos de fácil acceso.

A través de las encuestas las empresas pueden conocer la opinión de sus clientes, evaluar la preferencia de sus productos y analizar datos que contribuyan a la mejora empresarial y de servicios.

Investigación correlacional: este tipo de investigación relaciona dos variables para evaluar la interacción de un respecto a la otra. Permite establecer criterios entre dos grupos bien definidos, con la finalidad de dar valor a las relaciones naturales de las variables estudiadas.

Investigación experimental: es mayormente utilizada en las ciencias naturales, y consiste en analizar y verificar o no la validez de una teoría. Este tipo de investigación utiliza procesos y experimentos que se ajusten a una o varias teorías para analizar el comportamiento de las mismas y evaluar su veracidad.

En este capítulo se analizarán los aspectos fundamentales del marco teórico para investigaciones cuantitativas, se mostraran los criterios para realizar un marco teórico coherente y con sentido lógico adaptado de forma apropiada a la investigación cuantitativa. 
Implicaciones del problema en la investigación cuantitativa.

Para plantear el problema en la investigación cuantitativa, es necesario considerar los objetivos de la investigación, que serán la ruta a seguir o el horizonte del problema a resolver, lo que dará las pautas para que el trabajo se ejecute apropiadamente. Los objetivos de todo trabajo de investigación enfocan y dan las directrices a seguir para que se alcancen los resultados.

Además de los objetivos, es importante destacar la redacción del problema, pues no debe incluir información ambigua y debe ser conciso para definirse. Normalmente se describe como pregunta, para que además tenga la posibilidad ser verificado o comprobado a través de algún proceso de medición o validación.

Todo problema de investigación cuantitativa debe incluir una justificación clara y bien definida donde se establezcan los criterios que hacen posible la propuesta a desarrollar.

Definir una idea clara del problema permitirá reconocer cuáles son las limitaciones que conducen en la realización de la investigación, y que serán clave para establecer el alcance. Además deben tenerse en cuenta las delimitaciones que harán posible una investigación cuantitativa en el tiempo establecido, y permitirá definir los resultados de la investigación.

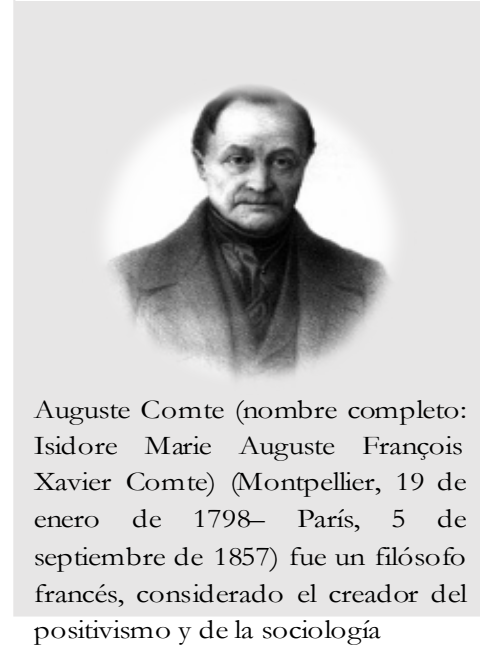

Una idea clara del problema permitirá conocer las limitaciones y delimitaciones del problema a estudiar, facilitando la focalización de los objetivos que se desean alcanzar.

Resulta importante en la investigación cuantitativa establecer una revisión bibliográfica que contextualice la investigación, que centre la investigación en torno a trabajos previos que aporten de manera significativa al estudio que se realiza. Este proceso de elaboración del estado del arte es fundamental para definir un planteamiento del problema bien focalizado, que permita conocer hasta dónde está el conocimiento actual de la temática de estudio, y que proyecte la investigación que se desea realizar.

Tomando en cuenta estos elementos, se plantea finalmente la propuesta que se va a realizar en el proceso de investigación cuantitativa, y se establecen los parámetros que harán posible la ejecución de la investigación.

En este apartado el grupo de investigación deberá formular una idea clara y concisa, que defina la propuesta de investigación con realce y sentido innovador para lograr un impacto científico apropiado.

La elaboración de un problema de investigación cuantitativa debe seguir los rigores científicos apropiados para asegurar una investigación ética y con resultados confiables.

En la figura 1 es posible visualizar los elementos asociados al planteamiento del problema en la investigación cuantitativa, se resume de forma gráfica lo antes descrito y permite a través de cuadros utilizar una guía didáctica para generar un problema de investigación cuantitativa. 


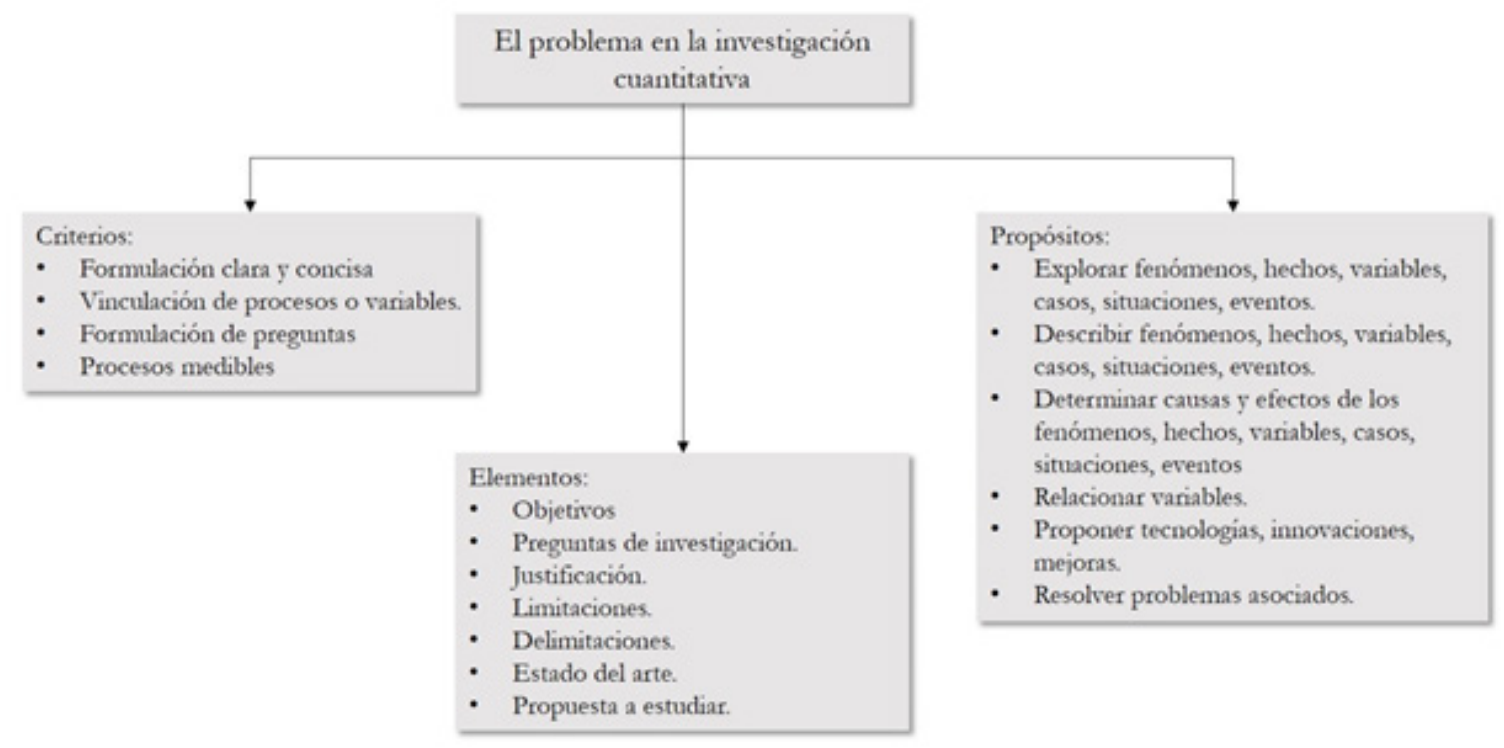

Figura 1. Aspectos del planteamiento de problema en la investigación cuantitativa.

Puede decirse entonces que el planteamiento del problema es definir y afinar los detalles de la investigación, es concretar las ideas y delimitar la investigación. Para lograr una buena afinidad del tema y una definición apropiada, es importante la revisión documental, que no solo aporta de manera significativa a la investigación, sino que además permite la contextualización del estudio y la validez de la innovación del estudio, pues permite evidenciar el aporte que la investigación ofrece a los trabajos ya realizados. Cuando la idea ha sido ya concebida y definida, es fundamental establecer los límites que harán posible el trabajo. La investigación debe centrarse únicamente a los intereses del equipo investigador, a los recursos, al tiempo disponible y a la finalidad del estudio.

En la investigación cuantitativa, por lo general, se busca probar hipótesis, estimar valores, definir variables o cantidades. Algunos autores [1] afirman que los planteamientos del problema en la investigación cuantitativa, están principalmente dirigidos a:

-Explorar hechos, poblaciones, variables, para cuantificarlos. Un ejemplo de ello es: indagar sobre las fallas eléctricas en equipos de alta potencia, evaluar las características de la diabetes en personas mayores, entre otras investigaciones de diferentes disciplinas que pueden entrar dentro del concepto cuantitativo.

-Evaluar casos o situaciones previas de situaciones actuales, esto permite hacer estadísticas y análisis numéricos de dichas situaciones para poder predecir conductas. Un ejemplo puede ser la evaluación de una enfermedad en distintas épocas y su evolución en el tiempo, también puede ser un ejemplo la evaluación de conductas sociales en distintas épocas, ante ciertas circunstancias, que permitan predecir situaciones futuras.

- Realizar contrastes o comparaciones entre situaciones, que permitan categorizar eventos, casos, hechos, variables. Esto puede ejemplificarse con los estudios realizados en torno a las elecciones presidenciales, también puede visualizarse en el estudio de un virus en grupos etarios, así como la comparación del uso de sensores en la medición de fluidos en tanques de crudo.

- Determinar las causas y las consecuencias de determinados fenómenos, es también una forma de plantear un problema en la investigación cuantitativa, pues permite conocer de forma numérica el impacto que una variable puede tener sobre otra. Un ejemplo de ello incluye el estudio sobre el impacto de las migraciones en la economía de los países, así como el impacto de la corrosión en las superficies expuestas.

-El desarrollo de tecnología, desarrollo de ingeniería, siempre es un problema cuantitativo. Que puede verse reflejado en el desarrollo de un brazo robótico para prótesis humana, así como el desarrollo de inteligencia 
artificial para el reconocimiento de emociones humanas, también el desarrollo de un software para enseñanza parvulario, entre otras aplicaciones cuantificables.

El planteamiento del problema en la investigación cuantitativa puede involucrar una combinación de áreas, combinación de variables, de casos, y por ende combinación de soluciones, que tienen la misma finalidad de comprobación de hipótesis. Existen criterios que definen el problema en la investigación cuantitativa, y estos deben ser claros y concisos. El planteamiento del problema no puede redactarse a la ligera, pues debe incluir interrogantes que harán posible focalizar la investigación. Estas preguntas deberán centrar las bases de la investigación y serán el eje principal del trabajo. Por ejemplo: ¿Cómo se relaciona la variable A con la variable B?, ¿De qué manera impacta la variable A sobre la variable B?, entre otras variadas preguntas que deben estar presentes para poder focalizar la investigación.

El planteamiento del problema debe ir dentro de las posibilidades de demostración bajo métodos empíricos, que permita la resolución de un problema, un caso, un hecho, y que este pueda ser verificable. Así pues, debe ser observable y debe permitir la evidencia de los fenómenos. Por ejemplo: Analizar el comportamiento de la luz solar en las plantas, es completamente verificable. Pero analizar el alma de los gatos, no es algo que puede ser observable. Plantear un problema de investigación cuantitativa, involucra conocer lo que se desea investigar, conocer los parámetros de la investigación, conocer todos los aspectos que harán posible la ejecución de la investigación y tener calidad de los recursos con que se cuenta, ya sean recursos humanos, materiales o de tiempo. Además es importante conocer los recursos científicos y tecnológicos con los que se cuenta para la ejecución de la investigación, los cuales deberán estar presentes dentro de los aspectos metodológicos del estudio.

\section{Aspectos a considerar para el planteamiento del problema en la investigación cuantitativa.}

La elaboración del planteamiento del problema en la investigación cuantitativa debe considerar algunos aspectos importantes que serán determinantes para definir la idea de investigación y su ejecución en el tiempo.

Antes de empezar la investigación los autores deberán primero qué desean investigar, y de qué manera podrían realizar este trabajo y además para qué sería necesario realizar este trabajo.

De tal manera que la investigación debe pensarse previamente, tal como un arquitecto piensa previamente qué tipo de vivienda desea realizar, o como una manicurista piensa qué tipo de diseño de uñas desea hacer. En todos los campos profesionales debe existir una concepción primaria de la idea para poder ejecutarla.

\section{Formulación de objetivos}

El primer paso para llevar a cabo un trabajo de investigación, deberá ser la formulación de los objetivos, que deben ir enfocados en el propósito o finalidad de la investigación. Los objetivos deben ser claros y concisos, deben ser medibles, deben ser factibles y realizables y no pueden permitir ambigüedades. Los objetivos deben cumplir con ciertos rigores para que puedan ser la guía a seguir en el proceso de investigación. Estos rigores incluyen la redacción, la congruencia y la afinidad de la investigación.

Los verbos a utilizar en los objetivos deben ir escritos en infinitivos [2], deben también ser definitivos de los hechos que se desean ejecutar, por ejemplo:

- Describir

- Analizar

-Estimar

-Determinar, entre otros.

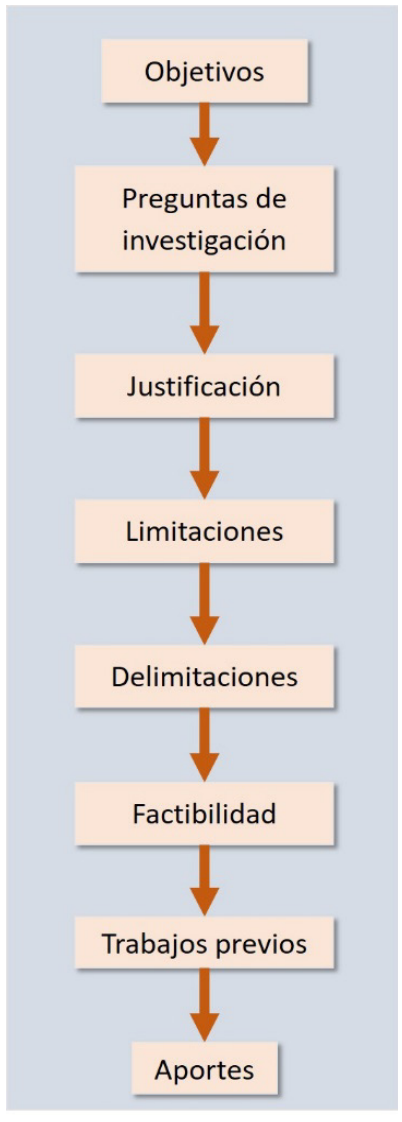

Figura 2. Elementos del planteamiento del problema. 
Los objetivos deben ser claros y concisos, deben especificar lo que se desea investigar y deben ser la guía durante todo el proceso de investigación

\section{Los objetivos deben vincularse entre sí, para poder darle coherencia a los resultados de la investigación.}

La definición de los objetivos de la investigación debe incluir dos categorías; el objetivo general y los objetivos específicos [3]. Estos deben ir relacionados entre sí, y deben contextualizar la situación que se desea atender. Así pues, el objetivo general debe enmarcar la meta macro de la investigación, lo que se desea alcanzar al final de todo el proceso. Mientras que los objetivos específicos deben focalizarse en las sub metas que conducen a la meta macro.

\section{Las preguntas de investigación}

Plantear la o las preguntas de investigación es fundamental para encaminar la investigación. Normalmente estas preguntas incluyen ¿Qué?, ¿Por qué?, ¿Cómo? Pero también pueden formularse desde otras perspectivas, como ¿Es posible que...? , ¿Es factible que...?, ¿Influye la... en la...? O entre otras que se ajustan a diferentes escenarios [4].

Las preguntas de investigación deben cumplir con ciertos criterios para poder incluirse efectivamente en el planteamiento del problema [1], [5], estos son:

Las preguntas de investigación deben responderse con facilidad, por tanto deben ser claras y concisas, de tal manera que en el proceso de investigación se dé respuesta a ellas con la misma claridad con la que fueron formuladas.

Las preguntas de investigación deben establecer los criterios de tiempo y espacio donde se ejecutará la investigación, de tal manera que incluyan los parámetros de años, meses, o especificidades temporales, así como los lugares o sitios donde se ejecutará el trabajo. También es posible que estos aspectos se incluyan en párrafos complementarios a las preguntas de investigación.

Las preguntas deben formularse de tal manera que puedan medirse, que puedan responderse a través de procesos o métodos concretos que definan la respuesta coherente y clara. Por tanto deben ser observables y reales.

A pesar de que las preguntas de que las preguntas de investigación deben poder responderse con claridad, estas no deben estar respondidas con anterioridad, lo que significa que las preguntas deben ser una incógnita real para los investigadores, de lo contrario la investigación no tendría sentido.

Las preguntas de investigación deben aportar significativamente a la comunidad científica, al área de investigación, a un área profesional.

Si las preguntas de investigación son ambiguas, no podrá darse una respuesta concisa de ella, y la investigación podría quedar inconclusa o sin resultados confiables [6]. Por tanto, las preguntas de investigación deben ser coherentes y claras [7]. Tampoco pueden redactarse las preguntas de investigación con demasiada generalidad, ya que de esta manera no podrán aportar nada concreto a la investigación. 


\section{Ejemplos de objetivos de investigación}

Para redactar los objetivos de la investigación, es importante distinguir según el tipo de trabajo que se desea realizar [1]:

Para trabajos exploratorios:

En estos casos es fundamental conocer los conceptos o variables que se involucran en el trabajo, así como el contexto donde se formulará la investigación. Una estructura apropiada podría ser:

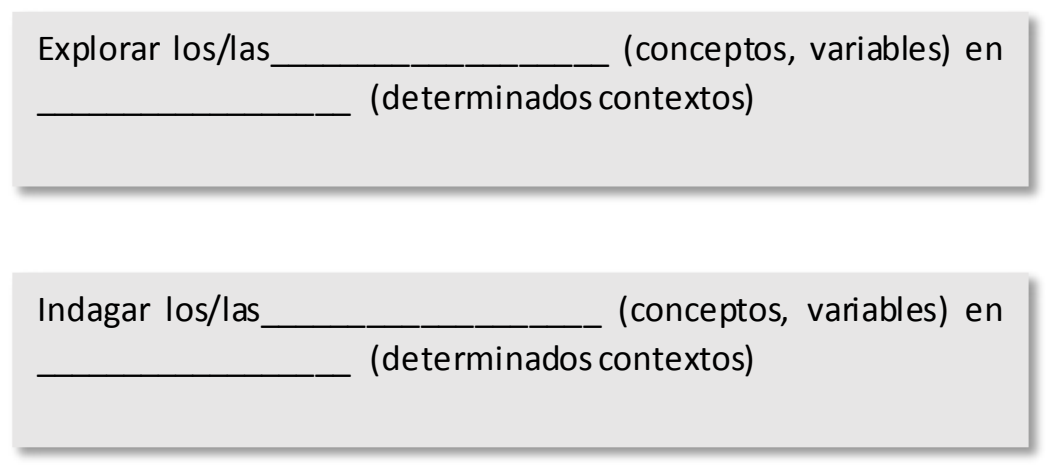

Para este tipo de investigación los verbos más comúnmente utilizados suelen ser: examinar, indagar, investigar, explorar y estudiar.

Para trabajos descriptivos:

Otro tipo de investigación es aquella que describe algún concepto, fenómeno o variable, y en este caso el objetivo del trabajo requiere otro tipo de redacción, que podría ser el siguiente:

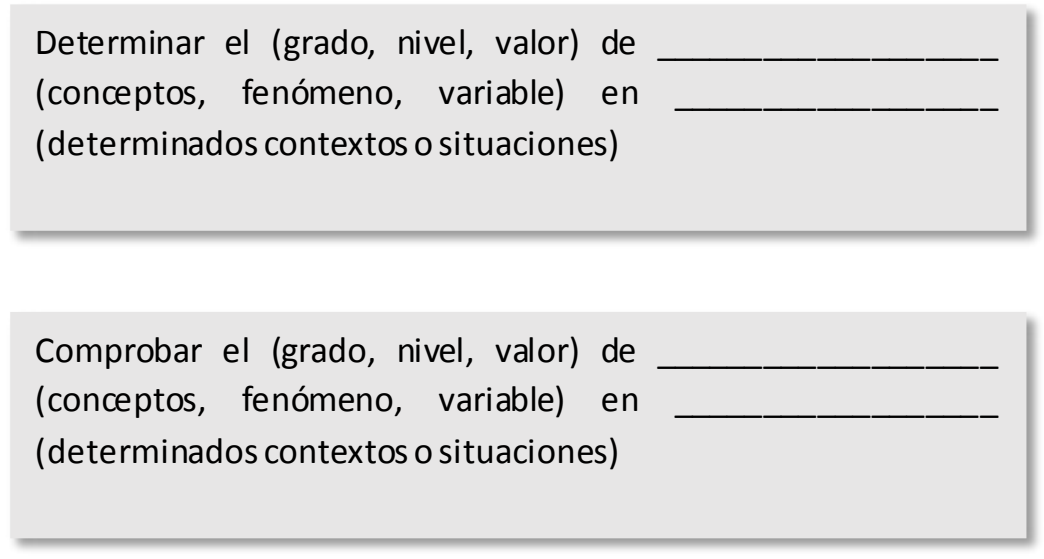

Para trabajos comparativos de variables:

Otro tipo de investigación es aquella que vincula dos o más variables para atender una situación. En estos casos, la redacción de objetivos debe especificar el vínculo de las variables y ser específico en lo que se desea alcanzar. Una estructura para ello podría ser:

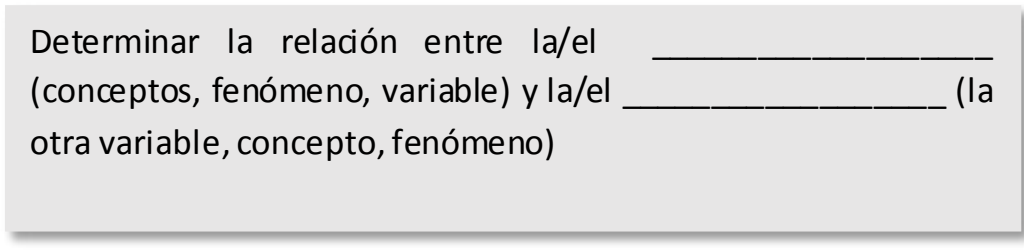




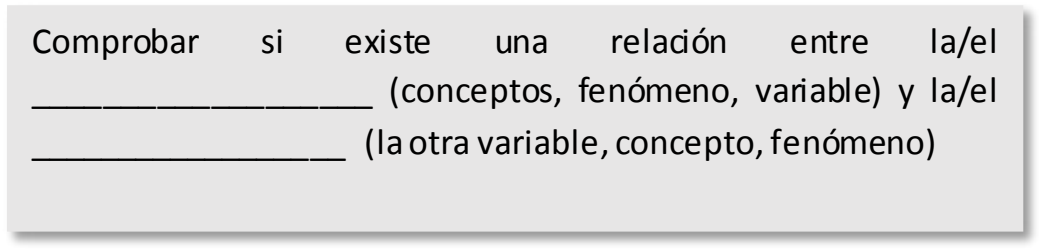

Algunos ejemplos podrían ser:

Ejemplo 1: Determinar el nivel de soda cáustica necesaria para la disolución de bauxita en el proceso de fabricación de alúmina.

Ejemplo 2: Comprobar el grado de satisfacción laboral en el personal docente durante el tiempo de pandemia.

Ejemplo 3: Determinar la relación entre los detalles afectivos y la estabilidad emocional de las parejas.

Ejemplo 4: Comprobar si existe una relación entre los estímulos estresores y la presencia de enfermedad en las personas.

\section{Ejemplos de preguntas de investigación}

Para formular las preguntas de investigación es importante que el investigador tenga muy clara la idea del proyecto y pueda internalizar cuáles son sus intenciones con el estudio. En el caso de los estudios exploratorios, es posible establecer las preguntas de investigación como siguen:

¿Cuáles son los orígenes, causas, principios de
conceptos, fenómenos) en_

¿Cuáles la/el (relación, nivel, grado) de_ (variables,
conceptos, fenómenos) en__ ? (un determinado contexto)

[1] afirma que este tipo de redacción plantea que la variable independiente tiene un efecto en la variable dependiente, por esto se dice que la causa origina una consecuencia.

Otra forma de redactar las preguntas de investigación, pueden seguir el siguiente formato:

¿Existe alguna relación significativa entre__
fenómeno o concepto) y_uariable, fenómeno o
concepto) en cuanto a__

Ejemplos de esto podrían ser:

Ejemplo 1: ¿Existe una relación significativa entre los estados emocionales y el desempeño laboral?

Ejemplo 2: ¿Cuál es el nivel de descargas parciales en máquinas de gran potencia en empresas hidroeléctricas?

Ejemplo 3: ¿Cuáles son los principios de funcionamiento del campo electromagnético que influyen en los trenes 
eléctricos?

Estos ejemplos son válidos en diferentes tipos de estudios, de una amplia variedad de áreas profesionales, donde se desee establecer criterios numéricos que permitan conocer más a fondo el fenómeno. Todas las preguntas de investigación cuantitativa conducen a algún tipo de recolección de datos numéricos, que además pueden complementarse con procesos estadísticos.

La formulación de preguntas de investigación puede resumirse en tres pasos esenciales:

Paso 1: Definir un tema de investigación

Esto comprende diversas situaciones, no necesariamente negativas. Una problemática de investigación puede ser una situación de felicidad o alegría, pero también una situación de enfermedad, un problema de automatización o una amplia variedad de situaciones en todas las áreas profesionales. Para conocer situaciones o problemas de estudio de nuestra área de investigación lo fundamental es leer, conocer los nuevos desarrollos, las nuevas tecnologías, nuevas propuestas, descubrir vacíos, huecos en las áreas de estudio.

Paso 2: Delimitar la situación de estudio.

Es necesario conocer con claridad lo que se desea investigar y a su vez conocer hasta dónde se llevará a cabo la investigación y cuáles serán los parámetros para establecer los límites del trabajo. Esta delimitación permitirá establecer lugares, contextos, grupos, tipos de variables, que caractericen el proyecto. Las preguntas de investigación deberán incluir aspectos concisos de la delimitación del problema.

Paso 3: Desglosar las estrategias del problema de estudio.

Este paso permitirá descubrir nuevas preguntas de investigación. Por ejemplo_

¿Por qué el nivel de estrés académico es alto en universidades públicas?, lo que podría sugerir otras preguntas como:

¿Influye la capacitación docente en el nivel de estrés académico en las universidades públicas?

¿Los aspectos económicos incluyen en el nivel de estrés académico en las universidades públicas?

También es posible generar preguntas de investigación si nos preguntamos por las consecuencias del problema de estudio:

¿Cómo puede afectar el estrés académico en el desempeño laboral?

Cuando pensamos en las posibles soluciones que tendría el problema, se abren otras opciones de interrogantes:

¿Reducir el número de horas laborales podría mejorar el estrés académico?

Si además se formulan inquietudes en torno a otros escenarios, entonces es posible conseguir otras aristas para las preguntas de investigación:

¿El estrés académico en universidades públicas es mayor en hombres que en mujeres?

También es posible incorporar circunstancias históricas del problema que conduzcan a visualizar otras opciones en la formulación de preguntas de investigación:

¿El estrés académico en universidades públicas ha sido una constante en los últimos veinte años?

La pregunta de investigación es la parte más importante para poder alcanzar el éxito de la investigación. 


\section{Marco teórico en la Investigación Cuantitativa}

El marco teórico es el conjunto de elementos teóricos que contextualizan el proyecto, esto incluye analizar y exponer enfoques y postulados teóricos, antecedentes que permiten darle forma a la investigación.

El marco teórico permite afinar detalles de la investigación, por tanto es un complemento del planteamiento del problema, y es quien le da fuerza a las ideas formuladas en este.

La construcción del marco teórico va a revelar el panorama del problema, por ende está principalmente condicionado a la revisión bibliográfica. La búsqueda de fuentes documentales es un paso fundamental para encontrar nuevas propuestas, nuevas formulaciones, o ideas de los aportes que nuestro trabajo puede ofrecer al mundo. La revisión bibliográfica siempre será la mejor manera de descubrir los vacíos o huecos existentes en las distintas áreas de estudio, que pueden conducirnos a formular investigaciones de alto impacto.

Las funciones del marco teórico están comprendidas en:

- Pulir el planteamiento del problema

-Afinar las ideas preconcebidas.

- Orientar la investigación.

- Justificar el estudio.

-Encontrar las delimitaciones del estudio.

- Descubrir los vacíos que queremos llenar.

- Generar nuevas propuestas de investigación.

La manera de redactar un marco teórico puede ser:

-Por temas y sub temas.

-Redacción generalizada

-Apoyo en diagramas y mapas mentales.

Sus formas de presentarse pueden ser:

-Por la generación de nuevas teorías

- Fundamentado en una o varias teorías.

-A través de hipótesis.

-Según los espacios de tiempo. 


\section{Elementos del marco teórico}

El marco teórico debe comprender los antecedentes, investigaciones previas, trabajos anteriores, teorías, que sustentan las hipótesis, los proyectos de investigación, los planteamientos de un problema.

Con estos elementos se busca respaldar la investigación y destacar el aporte novedoso y de innovación de la investigación.

También en el marco teórico se resalta el área de estudio y la disciplina a la que pertenece la investigación, argumentando los conceptos y variables que intervienen en el estudio, con las formulaciones teóricas que validan las propuestas, hipótesis, problemas que se platean.

Los principales elementos del marco teórico son:

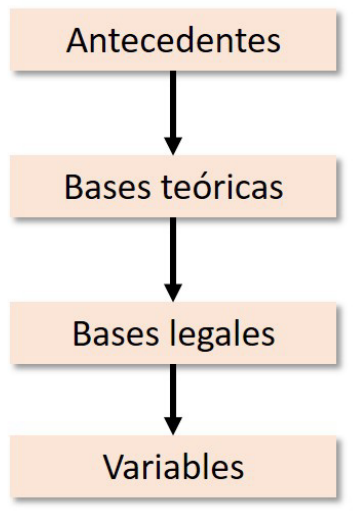

Figura 3. Parámetros del marco teórico.

Los antecedentes deben incluir los aspectos que contextualizan la investigación, que permiten conocer los avances anteriores y que permiten ubicar el problema en el presente. También permiten establecer una guía de trabajo con respecto a los procesos metodológicos y la información suministrada.

Por otro lado las bases teóricas se refieren a los aspectos conceptuales de la investigación, permiten establecer una fundamentación teórica del tema a investigar. En algunos casos es posible que la investigación sea completamente nueva y no existan teorías anteriores, pero deben existir parámetros para la formulación de una nueva teoría.

Los aspectos teóricos
permiten además aclarar el
tema de investigación,
definiendo las variables y
conceptos involucrados.

Es indispensable descubrir y alcanzar la argumentación teórica y toda la literatura relacionada con el planteamiento del problema.

Revisar exhaustivamente la
literatura para evaluar el
panorama del problema.


El marco teórico a pesar de indispensable en la investigación, no tiene una estructura única ni reglas únicas para su elaboración [8]. Sin embargo es posible orientarlo en torno a algunas ideas generales que pueden servir para la redacción apropiada del marco teórico.

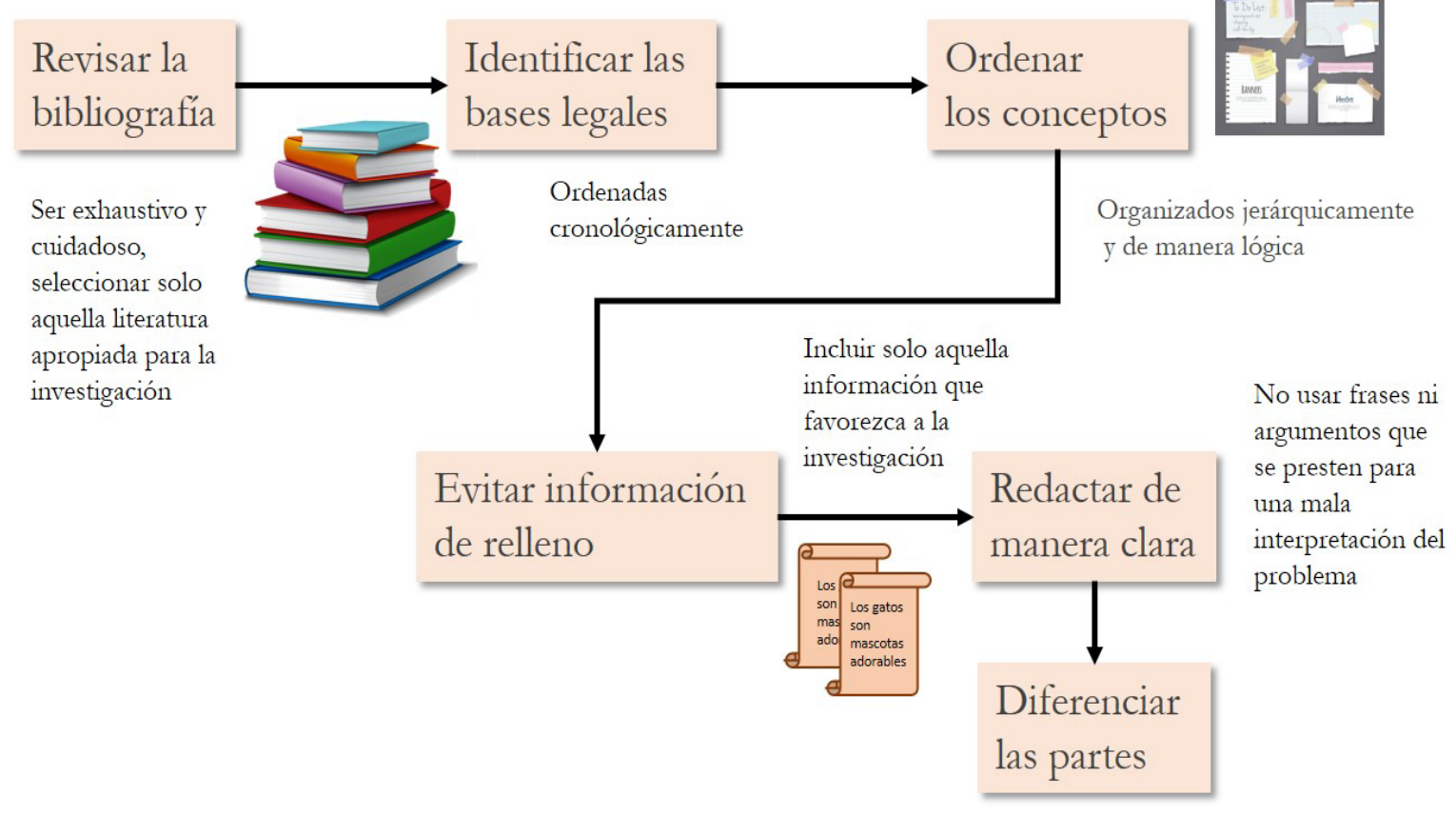

Las partes deben estar organizadas en títulos y subtítulos para que la información esté visible y entendible.

Figura 4. Aspectos a considerar para la elaboración del marco teórico.

Las partes básicas del marco teórico son la revisión bibliográfica, que involucra los antecedentes, luego es importante tener en cuenta las bases legales, que no aplica para todas los proyectos pero que si es importante para muchas investigaciones.

La organización de los conceptos debe ser clara y debe permitirle al lector ubicarse de forma armónica en el proyecto, de manera que se comprenda la generalización de las variables y definiciones, pero también las particularidades.

La inclusión de información de relleno es un error en la redacción del marco teórico, y puede ocasionar la desvaloración del trabajo, por ello es importante no desviarse del tema de estudio.

La redacción en todo momento debe tener claridad, sencillez y ser concisa, para evitar el uso de tecnicismos inapropiados, palabras rebuscadas y cualquier otro tipo de escritura que no favorezca la investigación [9].

Finalmente es importante que las partes que componen el marco teórico estén organizadas en títulos y subtítulos, con categorías apropiadas para diferenciar la relevancia de uno respecto del otro. El marco teórico no debe incluir sub capítulos, pero si secciones bien identificadas y organizadas para darle sentido a la contextualización del problema. 
Cuando se va a realizar la revisión bibliográfica, es importante considerar principalmente las revistas científicas, ya que ellas aportan información más reciente sobre los últimos avances en todas las disciplinas. Este tipo de fuentes contribuirá significativamente a la investigación. Para la selección de las revistas es necesario primeramente revisar aquellas que sean de acceso abierto, porque ofrecen artículos completos disponibles, que pueden aportar de manera relevante al proyecto.

Otro tipo de fuente confiable son los libros, sin embargo es importante resaltar que estos pueden tener información más clásica que debe seleccionarse cuidadosamente, pues en algunos casos la temática de estudio es muy cambiante y los libros pasan a tener información caducada de forma rápida.

Luego estas dos primeras fuentes confiables, es posible revisar otros tipos de fuentes, pero los autores deberán analizar muy bien la información y ser muy críticos para poder incluirla en su proyecto. Los block o páginas generales pudieran tener información errada, y pudieran además tener plagios o publicaciones sin permisos, no originales, y que pudieran perjudicar el trabajo de investigación.

El marco teórico también pudiese incluir aspectos de cálculo y que conlleve ecuaciones, según el tipo de investigación, y en estos casos los autores deberán asegurarse de realizar las ecuaciones con un gestor apropiado, ya que incluir ecuaciones en forma de gráficas le restará presencia y elegancia al trabajo y se prestará para pensar que los autores no hicieron una debida revisión de la literatura.

Los reportes de investigación son otro tipo de fuentes confiables para el marco teórico, ellos presentan los resultados y hallazgos de un conjunto de procesos y análisis de datos inmersos en un marco teórico previo. Estos permitirán encontrar ejemplos, formas de redacción, estructuras y argumentos científicos que puedan aportar al trabajo de investigación.

Algunos ejemplos de marco teórico se describen a continuación:

Ejemplo 1: Marco teórico en ciencias sociales

Tema: Desafíos y oportunidades de la inteligencia artificial en Latinoamérica.

Antecedentes: Se deberá consultar en trabajos científicos, tesis, ensayos, libros, sobre ¿qué es la inteligencia artificial?, ¿Cuáles autores han desarrollado trabajos de inteligencia artificial en Latinoamérica? ¿Cuál ha sido el impacto en los últimos años? Entre otras consultas que involucren la historia y el presente del tema de estudio [10]. Bases teóricas: Se deberá indagar sobre ¿cuáles son los desarrollos en la inteligencia artificial?, ¿de qué manera se ha desarrollado la inteligencia artificial en Latinoamérica?, ¿Cómo es el desarrollo económico en Latinoamérica y de qué manera influye esto en las nuevas tecnologías?, Aspectos legales de la investigación. Entre otras interrogantes que conducirán a contextualizar el problema de estudio.

Ejemplo 2: Marco teórico en ingeniería.

Tema: Automatización de planta de oxígeno en la Siderúrgica del Orinoco,

Antecedentes: Los autores deberán consultar fuentes confiables para determinar los aspectos y teorías relacionadas con la historia de las siderúrgicas, de qué manera han venido funcionado las plantas de oxígeno a lo largo del tiempo, cuáles autores han destacado trabajos similares.

Bases teóricas: Se deberá incluir información de fuentes confiables sobre ¿Qué es una siderúrgica?, ¿Qué papel juega la planta de oxígeno en la elaboración de acero?, ¿Cuáles son los elementos, equipos, variables, involucradas en el proceso de producción de acero?, entre otras muchas interrogantes asociadas al conocimiento de los paráme- 
tros que componen el tema de estudio.

Ejemplo 3: Marco teórico en educación.

Tema: La lectoescritura en niños de educación parvularia.

Antecedentes: Deberá revisarse la literatura en torno a ¿Cuáles trabajos se han desarrollado en torno al tema?, ¿De qué manera se ha venido dando la lectoescritura en la educación parvularia?, ¿Cómo ha venido influyendo el proceso de lectoescritura en el aprendizaje de los niños?, y todas las evaluaciones literarias que permitan hacer retrospectiva del problema y condicionar esa realidad para justificar el estudio presente.

Bases teóricas: Deberá incorporarse en este aspecto aquellos fundamentos teóricos de autores destacados en el área de educación y sus perspectivas para las mejoras de la enseñanza, así como aquellos conceptos sobre la lectoescritura y su importancia en el desarrollo de los niños.

En cualquiera de los ejemplos es importante reconocer los aportes de otros investigadores, así como utilizar la información existente para darle fortaleza al trabajo que queremos desarrollar. Ubicar rendijas o huecos en las investigaciones previas permitirá encontrar lo novedoso del estudio que deseamos desarrollar. La lectura selectiva de las fuentes bibliográficas es fundamental para desarrollar un proyecto de investigación innovador, de impacto y con una redacción apropiada.

[1] sugiere una estructura básica para el análisis y evaluación de las fuentes bibliográficas, que pueden servir para cualquier tipo de investigación, sea cuantitativa, cualitativa o mixta.

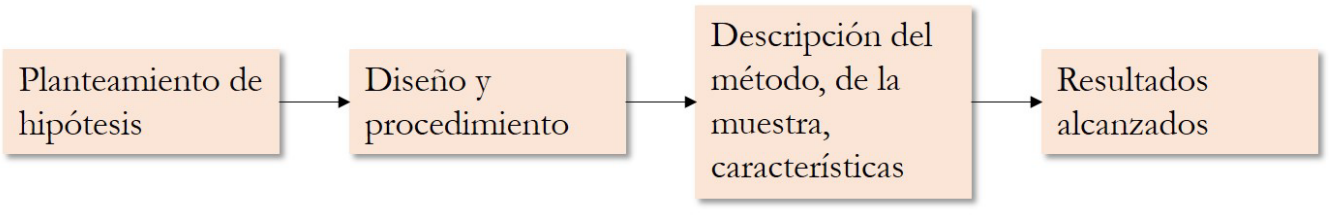

Figura 5. Elementos a considerar para la revisión bibliográfica.

Otra forma de presentar el marco teórico es a través de índices generales, que se irán desarrollando y afinando hasta lograr las especificidades necesarias para concretar las teorías de la investigación. Es importante resaltar que el marco teórico debe ajustarse a los antecedentes y a las novedades del problema de estudio, que serán empíricos, con datos, con números y que ello conducirá a la investigación cuantitativa.

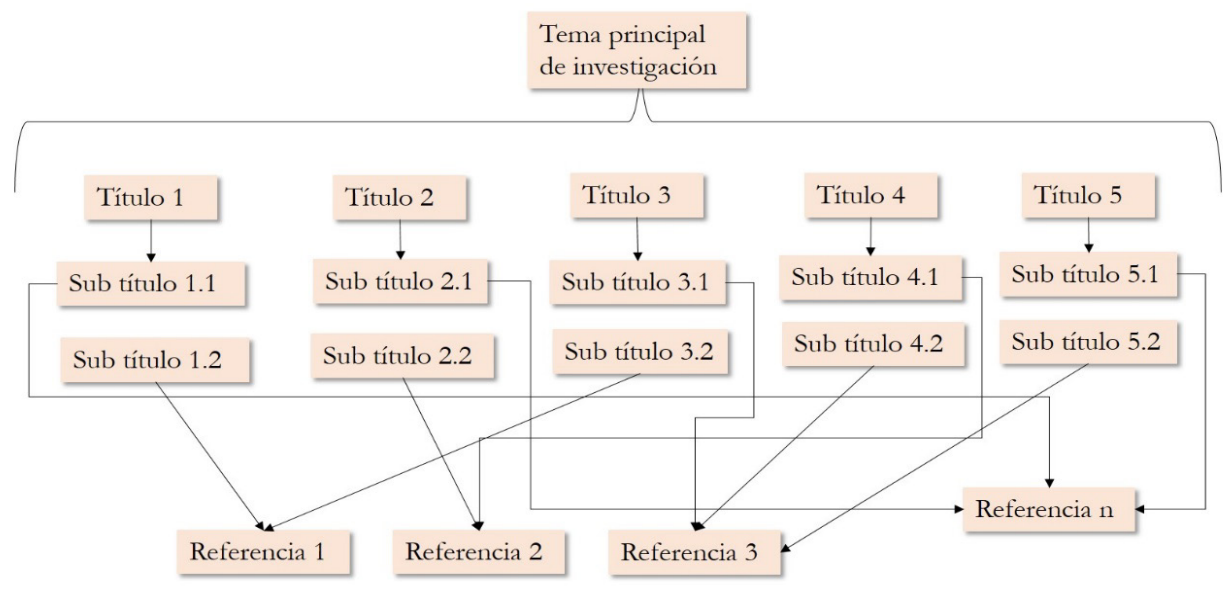

Figura 6. Estructura del marco teórico y su relación con las referencias 
Las referencias pueden relacionarse a uno o varios conceptos, así mismo es posible que un mismo concepto se relacione con una o varias referencias.

\section{Resumen del capítulo}

Finalmente en este capítulo se han tratado todos los aspectos necesarios para la formulación del planteamiento del problema y del marco teórico de una investigación cuantitativa, se han considerado los fundamentos teóricos y las posibles estructuras para realizar los capítulos preliminares de una investigación cuantitativa.

En un proceso de investigación cuantitativa deberán establecerse los parámetros planteados en este capítulo, con el fin de modelar una investigación apropiada de realce, pues la elaboración del planteamiento del problema resulta el eje medular de la investigación, la guía o el camino a seguir para el éxito de la investigación. Luego la incorporación del marco teórico apropiado establecerá los criterios de confianza del planteamiento del problema y será la base fundamental de la novedad de la investigación. 


\section{Referencias}

[1]R. Hernández-Sampieri, Metodología de la investigación, Mexico: Mc Graw Hill, 2018.

[2]L. Blaxter, C. Hughes y M. Tight, Cómo se hace una investigación., Barcelona: Gedisa, 2000.

[3]F. Alvira, «La perspectiva cualitativa y cuantitativa en las investigaciones sociales,» Revista estudios de psicología,

$\mathrm{n}^{\mathrm{o}} 11$, pp. 34-39, 1982.

[4]C. Monje Álvarez, Metodología de la Investigación Cuantitativa y Cualitativa,, Colombia: Universidad SurColombiana:

Facultad de Ciencias Sociales y Humanas, 2011.

[5]Briones, Métodos y Técnicas de Investigación para las Ciencias Sociales., Colombia: Colombia: Intituto

Colombiano para el Fomento de la educación suprior ICFES, 1996.

[6]M. C. Müggenburg Rodríguez y I. Pérez Cabrera, «Tipos de estudio en el enfoque de investigación cuantitativa,» Enfermería Universitaria, vol. 4, nº 1, p. 35.42, 2007.

[7]M. Amakforoosh, Metodología de la investigación., México, D.F.: Editorial Limusa, 2001.

[8]N. López y I. Sandoval, «Métodos y técnicas de investigación cuantitativa y cualitativa.,» [En línea].Available: http://recursos.udgvirtual.udg.mx/biblioteca/bitstream/20050101/1103/1/

Metodos_y_tecnicas_de_investigacion_cuantitativa_y_cualitativa.pdf. [Último acceso: 09 noviembre 2020].

[9]J. Argibay, «Muestra en investigación cuantitativa,» Subjetividad y procesos cognitivos, vol. 13, nº 1, pp. 13-29, 2009.

[10]M. Canales Cerón, Metodologías de investigación social, 1ª ed. - Santiago: Lom Ediciones,, 2006. 